\title{
Pembrolizumab-Induced Severe Neuropathy in a Patient with Metastatic Urothelial Carcinoma after Achieving Complete Response: Guillain-Barré Syndrome-Like Onset
}

\author{
Shuntaro Aokia, Masato Yasui a, b Hironao Tajirika ${ }^{a}$ Hideyuki Terao a \\ Makoto Funahashi ${ }^{a}$ Junichi Ohta ${ }^{a}$ \\ aDepartment of Urology, Yokohama Municipal Citizen's Hospital, Yokohama, Japan; \\ ${ }^{b}$ Department of Urology and Renal Transplantation, Yokohama City University Medical \\ Center, Yokohama, Japan
}

\section{Keywords}

Pembrolizumab · Methylprednisolone · Peripheral neuropathy · Guillain-Barré syndrome

\section{Abstract}

An 85-year-old female was admitted to our hospital for left ureteral cancer and para-aortic lymph node metastasis. To control hematuria, a laparoscopic retroperitoneal nephroureterectomy was performed, and papillary urothelial carcinoma (pT3b) was found. To treat paraaortic lymph node metastasis, she received chemotherapy with gemcitabine and nedaplatin. After 2 cycles, a computed tomography scan revealed its disappearance; however, bilateral lung metastases appeared. The patient was administered second-line therapy with pembrolizumab every 3 weeks. After 3 courses, lung metastases disappeared and she achieved a complete response. After the fifth administration of pembrolizumab, she was readmitted with right upper limb pain and weakness in both lower extremities. She was diagnosed with pembrolizumab-induced grade 3 peripheral neuropathy with Guillain-Barré syndrome-like onset. High-dose monocorticotherapy was initiated for treatment. Three weeks later, the pain and weakness of the limbs improved. After discharge, the dose of prednisolone was tapered and there was no relapse of adverse events. Pembrolizumab was discontinued at the onset of neuropathy, but she maintained a complete response. 


\section{Introduction}

Pembrolizumab, a humanized IgG4 monoclonal antibody, binds to programmed cell death-1 (PD-1) and directly inhibits the binding of PD-1 to programmed cell death-ligand 1 (PD-L1) and PD-L2 [1]. The PD-1 pathway, in which PD-1 binds to PD-L1, inhibits the activation and function of autoreactive $\mathrm{T}$ cells and promotes the development and function of regulatory T cells (Tregs). Via the expression of PD-L1 on its surface, which induces and maintains Tregs in the tumor microenvironment, tumor cells augment the suppression of antitumor T-cell responses and allow tumor progression. Pembrolizumab is used to treat several cancers. The antitumor effect of pembrolizumab is achieved by the blockade of the PD-1 pathway, which promotes immunity and leads to a variety of immune-related adverse events (irAEs) [2]. However, neurological AEs after anti-PD-1 therapy are rare [3].

Currently, there are no reports about neurological irAEs caused by pembrolizumab in the treatment of urothelial carcinoma. Herein, we report a patient with metastatic urothelial carcinoma who was treated with pembrolizumab, which led to severe Guillain-Barré syndrome (GBS)-like neuropathy.

\section{Case Presentation}

An 85-year-old female was admitted to our department with hematuria. A computed tomography (CT) scan showed a left ureteral mass lesion and para-aortic lymphadenopathy (Fig. 1a, b). Retrograde pyelography was performed and revealed a contrast defect and class V urine cytology (Fig. 1c). She was diagnosed with clinical Stage IV ureter cancer. However, hematuria was persistent and, to control local symptoms, we performed a laparoscopic retroperitoneal nephroureterectomy. The pathological results showed papillary urothelial carcinoma (pT3b). After surgery, she was administered systemic chemotherapy with intravenous gemcitabine plus nedaplatin, owing to her worsened renal dysfunction. After 2 cycles, a CT scan showed the disappearance of lymph node metastasis, but bilateral lung metastases appeared (Fig. 2a). Her treatment was changed to pembrolizumab (200 mg) every 3 weeks. After 3 cycles, the bilateral lung metastasis disappeared, detected by a CT scan, and the patient achieved a complete response (Fig. 2 b).

Three weeks after the fifth administration of pembrolizumab, she was readmitted with right upper limb pain and weakness in both lower extremities. In a blood test, the level of C-reactive protein was elevated to $8.6 \mathrm{mg} / \mathrm{dL}$, but there were no electrolyte abnormalities or thyroid dysfunctions that could cause neurological symptoms. Pyuria was detected and a CT
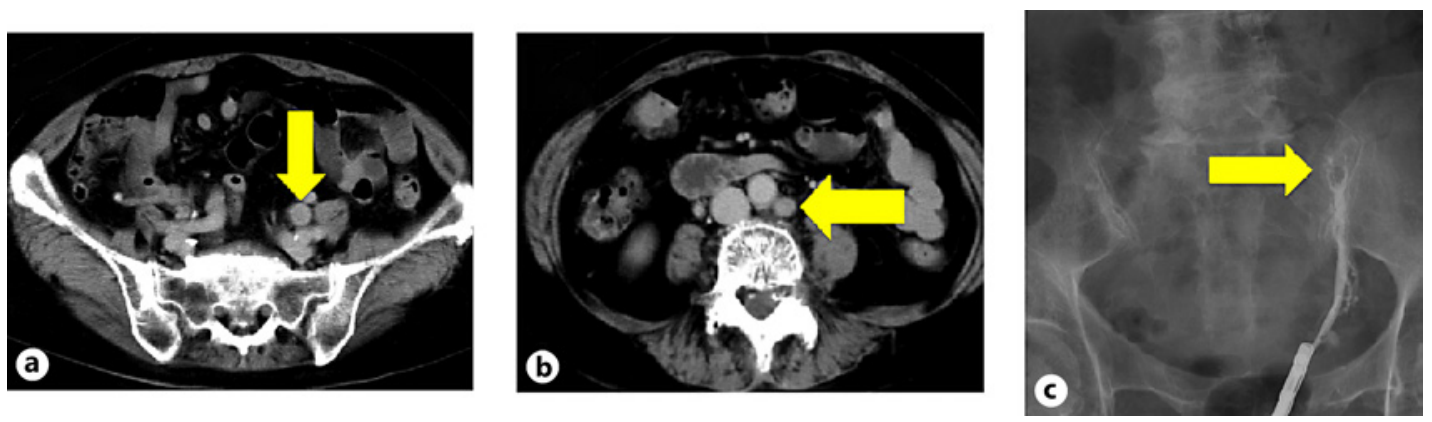

Fig. 1. a, b Abdominal enhanced computed tomographic images taken before surgery. A left ureteral mass lesion (a) and para-aortic lymphadenopathy (b). c Retrograde pyelography showing a contrast defect in the ureter. 
Fig. 2. a After 2 courses of gemcitabine and nedaplatin, a computed tomography scan showed the disappearance of lymph node metastasis, but bilateral lung metastases appeared. b After 3 courses of pembrolizumab, bilateral lung metastasis disappeared and the patient achieved a complete response.
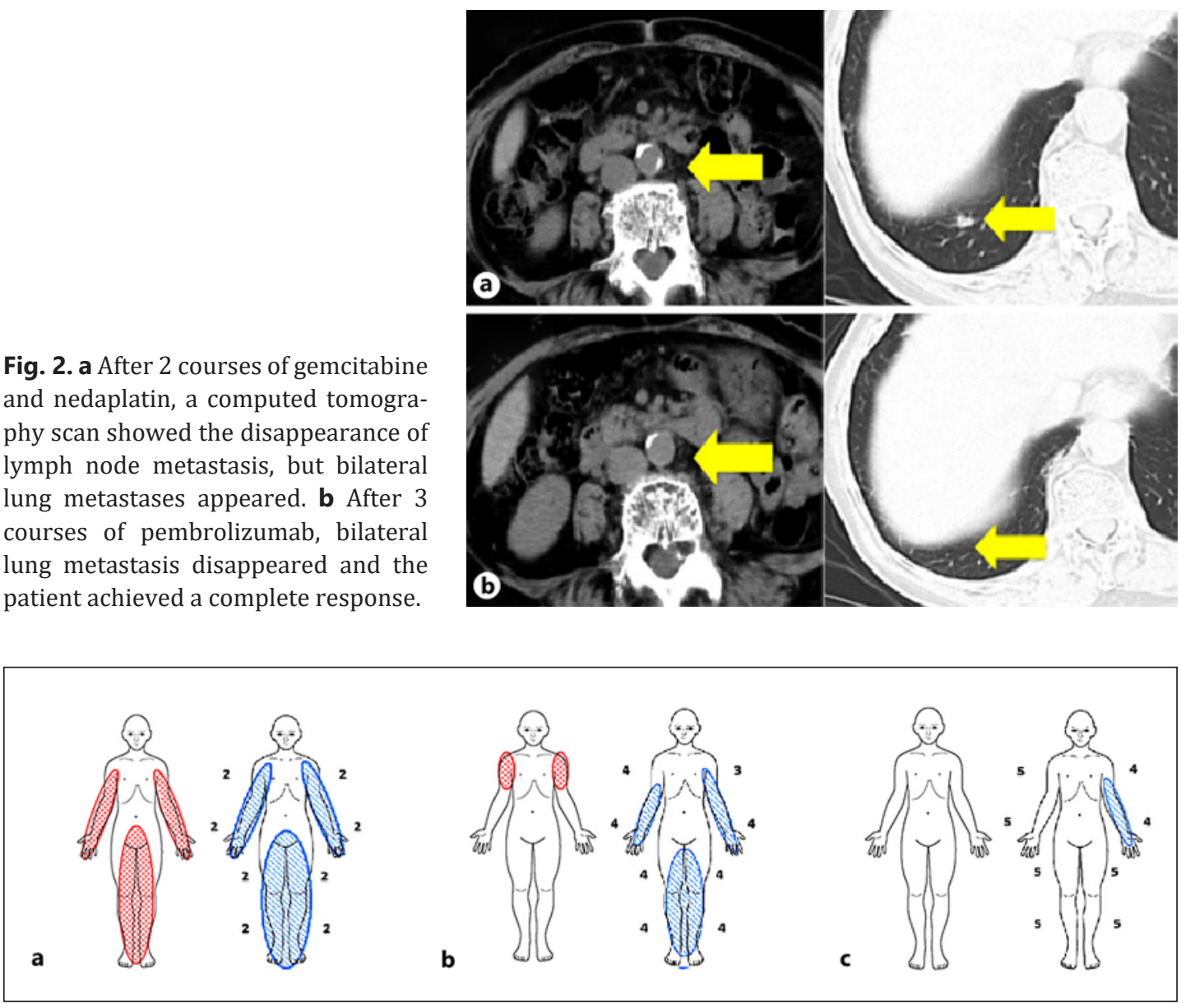

Fig. 3. Pain range (left) and weakness range (right). In the right picture, the numbers around the body represent manual muscle testing (MMT) scores of the biceps, brachioradialis, quadriceps, and anterior tibialis. a Immediately after admission, she could not move by herself because of strong pain and weakness. b One week after corticotherapy started, pain in the limbs improved significantly and MMT level recovered to 4. c At discharge, although weakness remained slightly in the left forearm, she could walk independently.

scan revealed pyelonephritis; thus, she started a course of antibiotics and pembrolizumab treatment was discontinued.

Immediately after admission, pain in the lower and left upper limbs appeared, becoming so strong that analgesics were ineffective. Because of the pain and weakness in the limbs, she could not lift her extremities and move by herself; her manual muscle testing score was 2 (Fig. 3a). We consulted the Department of Neurology and neurological examinations were performed. Cerebrospinal fluid analysis showed albuminocytological dissociation and nerve conduction studies revealed axonal disorder and radiculopathy. These results were similar to those observed in patients with GBS; thus, the diagnosis was pembrolizumab-induced peripheral neurology (grade 3) with a GBS-like onset pattern.

After the diagnosis of neuropathy, high-dose corticotherapy $(80 \mathrm{mg} /$ day of methylprednisolone) was administered. One day after treatment, limb pain improved and, 1 week later, neurological symptoms improved dramatically. Pain in the limbs had almost disappeared and she was able to move her limbs by herself; her manual muscle testing score recovered to 4 (Fig. 3b). Corticosteroids were gradually tapered to $30 \mathrm{mg} /$ day of prednisolone. Three weeks 
after admission, mild weakness in the limbs remained, but she was able to walk independently and was discharged (Fig. 3c).

The dose of prednisolone was gradually reduced at intervals of approximately 2 weeks and there was no relapse of neurological symptoms and other AEs. Prednisolone was reduced to $3 \mathrm{mg}$ /day 5 months after corticotherapy started. Pembrolizumab has been discontinued since the onset of neuropathy, but she maintained a complete response, which was detected by a CT scan after 6 months.

\section{Discussion}

The mechanism that underlies irAEs is unknown, but the blockade of the PD-1 pathway, which augments T-cell activation and promotes autoimmunity, may be involved [4]. Neurological AEs caused by anti-PD-1 therapy are rare, unpredictable, evolving, and life-threatening [3].

GBS, an inflammatory polyneuropathy, is associated with an autoimmune reaction to gangliosides. GBS mainly occurs after infection, which suggests that an aberrant autoimmune response damages peripheral nerves and their spinal roots [5]. Idiopathic GBS is treated with intravenous immunoglobulin or plasma exchange; however, monocorticotherapy is ineffective for the treatment of idiopathic GBS [6]. Corticosteroids are effective for the treatment of immune checkpoint inhibitor-induced GBS [7] and immune checkpoint inhibitor-induced GBS in a melanoma patient has been improved by monocorticotherapy [8]. Our case was also treated with monocorticotherapy and her symptoms improved dramatically. This suggests that the pathophysiology of immune checkpoint inhibitor-induced GBS is different from that of idiopathic GBS. However, monocorticotherapy may lead to unfavorable outcomes in patients with PD-1 inhibitor-associated neuromuscular complications [9]; thus, additional investigations are needed for the accurate evaluation of the pathophysiology of GBS.

Whether immunological activation by immune checkpoint therapy correlates with antitumor immunity has not been clarified. However, some studies suggest that irAEs correlate with the efficacy of immune checkpoint therapy [10-12]. Our patient maintained a complete response without relapse of neuropathy during pembrolizumab withdrawal. This suggests that there was a relationship between urothelial carcinoma and the efficacy of immune checkpoint therapy. There is no consensus about restarting immune checkpoint therapy after a severe irAE, because the permanent withdrawal of the immune checkpoint inhibitor after a severe irAE is often required and prospective clinical trials are limited. In this case, we decided not to resume pembrolizumab as no sign of recurrence has been detected. Whether to restart immune checkpoint therapy after another severe irAE depends on the judgment of clinicians, but it is a contraindication to resume immune checkpoint therapy after a life-threatening irAE [3].

\section{Conclusion}

We report a case of severe pembrolizumab-induced neuropathy in a patient with metastatic urothelial carcinoma. A complete response was maintained after pembrolizumab withdrawal, which suggests a relationship between irAEs and the efficacy of pembrolizumab. Physicians should be aware of the potential AEs of this antibody to inform the use of pembrolizumab, despite its anticancer efficacy.

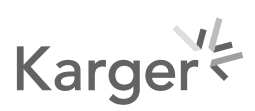




\section{Case Reports in Oncology}

Case Rep Oncol 2020;13:1490-1494

\begin{tabular}{l|l}
\hline DOI: $10.1159 / 000511567$ & ○ 2020 The Author(s). Published by S. Karger AG, Basel \\
\hline
\end{tabular} www.karger.com/cro

Aoki et al.: Pembrolizumab-Induced Neuropathy

\section{Statement of Ethics}

Written informed consent was obtained from the patient for publication of this case report and any accompanying images.

\section{Conflict of Interest Statement}

The authors declare no conflict of interest.

\section{Funding Sources}

None.

\section{Author Contributions}

Shuntaro Aoki, Masato Yasui, and Junichi Ohta contributed to the conception and design of the study; drafting and critical revision of the manuscript. Hironao Tajirika, Hideyuki Terao, and Makoto Funahashi contributed by final approval of the version to be published.

\section{Data Availability}

Owing to ethical restrictions, the raw data underlying this paper are available upon request to the corresponding author.

\section{References}

1 McDermott J, Jimeno A. Pembrolizumab: PD-1 inhibition as a therapeutic strategy in cancer. Drugs Today. 2015;51(1):7-20.

2 Francisco LM, Sage PT, Sharpe AH. The PD-1 pathway in tolerance and autoimmunity. Immunol Rev. 2010; 236:219-42.

3 Kao JC, Liao B, Markovic SN, Klein CJ, Naddaf E, Staff NP, et al. Neurological complications associated with antiprogrammed death 1 (PD-1) antibodies. JAMA Neurol. 2017;74(10):1216-22.

4 Postow MA, Sidlow R, Hellmann MD. Immune-related adverse events associated with immune checkpoint blockade. N Engl J Med. 2018;378(2):158-68.

5 Hughes RA, Cornblath DR. Guillain-Barré syndrome. Lancet. 2005;366(9497):1653-66.

6 Hughes RA, Brassington R, Gunn AA, van Doorn PA. Corticosteroids for Guillain-Barré syndrome. Cochrane Database Syst Rev. 2016;10:CD001446.

7 Brahmer JR, Lacchetti C, Schneider BJ, Atkins MB, Brassil KJ, Caterino JM, et al. Management of immune-related adverse events in patients treated with immune checkpoint inhibitor therapy: American Society of Clinical Oncology Clinical Practice Guideline. J Clin Oncol. 2018;36(17):1714-68.

8 Wilgenhof S, Neyns B. Anti-CTLA-4 antibody-induced Guillain-Barré syndrome in a melanoma patient. Ann Oncol. 2011;22(4):991-3.

9 Kao JC, Brickshawana A, Liewluck T. Neuromuscular complications of programmed cell death-1 (PD-1) inhibitors. Curr Neurol Neurosci Rep. 2018;18(10):63.

10 Rogado J, Sánchez-Torres JM, Romero-Laorden N, Ballesteros AI, Pacheco-Barcia V, Ramos-Leví A, et al. Immune-related adverse events predict the therapeutic efficacy of anti-PD-1 antibodies in cancer patients. Eur J Cancer. 2019;109:21-7.

11 Haratani K, Hayashi H, Chiba Y, Kudo K, Yonesaka K, Kato R, et al. Association of immune-related adverse events with nivolumab efficacy in non-small-cell lung cancer. JAMA Oncol. 2018;4(3):374-8.

12 Sanlorenzo M, Vujic I, Daud A, Algazi A, Gubens M, Luna SA, et al. Pembrolizumab cutaneous adverse events and their association with disease progression. JAMA Dermatol. 2015;151(11):1206-12. 